\title{
Percepção da qualidade de vida de indivíduos portadores de DCNT durante o período de isolamento social
}

\author{
Perception of the quality of life of individuals with DCNT during the period of social isolation \\ Percepción de la calidad de vida de las personas con DCNT durante el período de aislamiento social
}

Recebido: 13/10/2021 | Revisado: 22/10//2021 | Aceito: 16/11/2021 | Publicado: 25/11/2021

Júlia Carolina Lopes Silva

ORCID: https://orcid.org/0000-0002-9682-6581

Universidade Federal de Pernambuco, Brasil E-mail: julia.lopes@ufpe.br

Matheus Luis Cobel de Santana

ORCID: https://orcid.org/0000-0002-8929-0950

Faculdade de Comunicação Tecnologia e Turismo de Olinda, Brasil

E-mail: matheusluis90@gmail.com

Sarah Évelin Dias Campos

ORCID: https://orcid.org/0000-0003-2461-400X

Faculdade de Comunicação Tecnologia e Turismo de Olinda, Brasil

E-mail: saarah_dias@outlook.com

André dos Santos Costa

ORCID: https://orcid.org/0000-0001-5301-2572

Universidade Federal de Pernambuco, Brasil

E-mail: andre.santoscosta@ufpe.br

Jakeline Olindina Francelino

ORCID: https://orcid.org/0000-0002-9851-5269

Universidade Federal de Pernambuco, Brasil

E-mail: jakelineolindina.prof@hotmail.com

\begin{abstract}
Resumo
O estudo objetivou avaliar a percepção da qualidade de vida de indivíduos portadores de DCNT durante o período de isolamento social, sendo assim, trata-se de um estudo observacional, transversal, com amostra de 17 indivíduos, onde os participantes realizaram o Questionário de Qualidade de Vida SF-36, assim como uma Anamnese Nutricional, para levantar dados acerca da população, e um Recordatório 24 Horas, para evidenciar o consumo dietético da amostra. O estudo obteve aprovação do Comitê de Ética em Pesquisa. A partir da avaliação do questionário SF-36 foi possível observar que nenhum dos domínios apresentou média de escore superior, caracterizando uma qualidade de vida mediana à baixa, destacando principalmente os domínios Dor e Limitações por Aspectos Emocionais, evidenciando a fragilidade gerada pelo isolamento social e as adaptações que foram realizadas, levando a prejuízos em outros domínios como Vitalidade, Estado Geral de Saúde e Saúde Mental, dito isto, o isolamento social realizado em função da pandemia do Covid-19 se mostrou um fator principal na piora da qualidade de vida de indivíduos portadores de DCNT, população mais afetada, por se enquadrar como grupo de risco.
\end{abstract}

Palavras-chave: Qualidade de vida; DCNT; Isolamento social; Mídias digitais.

\begin{abstract}
The study aimed to evaluate the perception of the quality of life of individuals with CNCDs during the period of social isolation, therefore, it is an observational, cross-sectional study, with a sample of 17 individuals, where the participants took the Quality of Life Questionnaire. Vida SF-36, as well as a Nutritional Anamnesis, to collect data about the population, and a 24-Hour Recall, to show the sample's dietary consumption. The study was approved by the Research Ethics Committee. From the evaluation of the SF-36 questionnaire, it was possible to observe that none of the domains had a higher average score, characterizing a low to medium quality of life, mainly highlighting the Pain and Limitations due to Emotional Aspects domains, showing the fragility generated by social isolation and the adaptations that were made, leading to losses in other domains such as Vitality, General Health and Mental Health, that being said, the social isolation carried out due to the Covid-19 pandemic proved to be a major factor in worsening the quality of life of individuals with NCDs, the most affected population, as it fits as a risk group.
\end{abstract}

Keywords: Quality of life; DCNT's; Social isolation; Digital media.

\section{Resumen}

El estudio tuvo como objetivo evaluar la percepción de la calidad de vida de las personas con DCNT durante el período de aislamiento social, por lo que se trata de un estudio observacional, transversal, con una muestra de 17 
individuos, donde los participantes completaron el Cuestionario de Calidad de Vida SF-36, así como una Anamnesis Nutricional, para recopilar datos sobre la población, y un recordatorio de 24 horas, para evidenciar la ingesta dietética de la muestra. El estudio fue aprobado por el Comité de Ética en Investigación. A partir de la evaluación del cuestionario SF-36, se pudo observar que ninguno de los dominios presentó un puntaje promedio más alto, caracterizando una calidad de vida media a baja, destacando principalmente los dominios Dolor y Limitaciones por Aspectos Emocionales, mostrando la fragilidad generado por el aislamiento social y las adaptaciones que se realizaron, lo que provocó pérdidas en otros dominios como la vitalidad, la salud general y la salud mental, habiendo dicho que el aislamiento social causado por la pandemia Covid-19 resultó ser un factor principal en el agravamiento de la calidad de vida de las personas con DCNT, la población más afectada, porque encajan como grupo de riesgo.

Palabras clave: Calidad de vida; DCNT; Aislamiento social; Medios digitales.

\section{Introdução}

De acordo com a Organização Mundial de Saúde (OMS) a saúde não é apenas a ausência de doença, mas sim, um complexo formado por bem-estar físico, mental e social do indivíduo, sendo a qualidade de vida o resultado geral de todos esses fatores (Testa \& Simonson, 1996).

A associação de doenças crônicas com a qualidade de vida é um desafio para os profissionais de saúde, assim como para os indivíduos portadores de Doenças Crônicas Não-Transmissíveis (DCNT) e seus familiares, entretanto, esta discussão tem ganhado maior notoriedade, onde o avanço tecnológico da medicina, com o desenvolvimento de novos fármacos, elevou a relevância do tema, uma vez que este avanço tecnológico pode influenciar na qualidade de vida (Pierin, 2004; Silva et al., 2005).

Considerando que a qualidade de vida é resultado de outros fatores, estes são essenciais para se avaliar o indicativo de bem-estar na vida destes indivíduos, tais como: controle cognitivo, aspectos socioeconômicos, longevidade, saúde mental e produtividade (Somchida \& Fernandes, 2003).

Para os indivíduos portadores de DCNT a avaliação da qualidade de vida se torna ainda mais relevante, onde esta avaliação pode nos demonstrar o impacto dos tratamentos, assim como o impacto da doença nas atividades diárias do indivíduo, além da possibilidade de melhoria dos cuidados do paciente, através da avaliação das preferências do indivíduo de acordo com o tratamento ao qual ele está sendo exposto (Taylor, 1999; Fletcher, 1995).

Sendo assim, instrumentos de avaliação da qualidade de vida como o 36-Item Short-Form Health Survey (SF-36) são amplamente aplicados, podendo ainda ser utilizadas estratégias para utilização do instrumento, como: aplicação do questionário através de mídias digitais e realização de pequenas modificações na estrutura do questionário, para facilitar sua aplicabilidade, onde, mesmo apresentando questões em aberto, o SF-36 tem se mostrado como uma medida confiável e válida (Mahony, 1998; Walters, 2001).

Considerando que a literatura científica é escassa em dados que demonstrem o perfil da qualidade de vida durante o período de isolamento social ocasionado pela pandemia do Covid-19, buscamos com este estudo avaliar a percepção da qualidade de vida de indivíduos portadores de DCNT, durante o período de isolamento social.

\section{Metodologia}

Foi realizado Trata-se de um estudo quali e quantitativo, descritivo e transversal (Thomas et al., 2012) onde toda a coleta ocorreu de maneira online, e por contato telefônico. A realização do estudo se deu a partir da autorização do Comitê de Ética em Pesquisa da Faculdade de Medicina de Olinda, com o CAAE: 31644120.1.0000.8033.

A amostra, composta por 17 indivíduos, foi selecionada através da divulgação do projeto nas mídias sociais e recebeu, via WhatsApp, o Termo de Consentimento Livre e Esclarecido, onde o mesmo foi devidamente preenchido através do domínio Google Forms antes de serem encaminhados para os grupos de WhatsApp, para iniciar os protocolos de coleta.

Para análise da qualidade de vida foi utilizada a versão brasileira do Questionário de Qualidade de Vida SF-36, 
desenvolvido por Ware e Sherbourne, 1992, constituído por 36 itens que abordam 8 domínios, sendo eles: capacidade funcional (CF), limitação por aspectos físicos (LAF), dor (DOR), estado geral de saúde (EGS), vitalidade (VIT), aspectos sociais (AS), limitações por aspectos emocionais (LAE) e saúde mental (SM). O questionário foi posteriormente traduzido e validado para sua versão brasileira por Ciconelli (2011). Para nosso estudo o questionário foi transcrito para o domínio do Google Forms, e posteriormente distribuído para preenchimento dos participantes.

Para avaliação geral dos participantes, foi elaborada uma Anamnese própria para o estudo, onde foram levantados dados como: Autocuidado, realização de atividade física, nível socioeconômico e hábitos durante o isolamento social, fornecendo dados relevantes acerca da realidade dos participantes do estudo.

Também foi realizada a coleta do Relatório 24 Horas (R24h), instrumento que fornece dados qualitativos e quantitativos acerca da alimentação realizada pelo participante 24 horas atrás. Para realização do R24h foram realizados telefonemas para os participantes, onde os pesquisadores do projeto realizaram a entrevista e registraram em planilha do Excel.

\section{Resultados}

A amostra foi composta por 17 indivíduos e na análise descritiva obtivemos os seguintes dados: Quanto ao gênero, obtivemos público feminino, com $n=14$ (82,4\%), e masculino, com $n=3$ (17,6\%), e média de $1,18 \pm 0,39$, denominando um público majoritariamente feminino. Quanto a idade, obtivemos o mínimo de 26 anos e máximo de 77 anos, com uma média de $44,35 \pm 13,95$.

Em avaliação de escolaridade (Figura 1), evidenciamos que 11,8\% estudaram da $1^{\mathrm{a}}$ à $4^{\mathrm{a}}$ série do ensino fundamental; $5,9 \%$ estudaram da $5^{\mathrm{a}}$ à $8^{\mathrm{a}}$ série do ensino fundamental; 41,2\% têm o ensino médio completo e $41,2 \%$ têm o ensino superior completo, caracterizando assim um público majoritariamente escolarizado.

Figura 1. Grau de escolaridade dos participantes do estudo. Olinda, PE. Brasil.

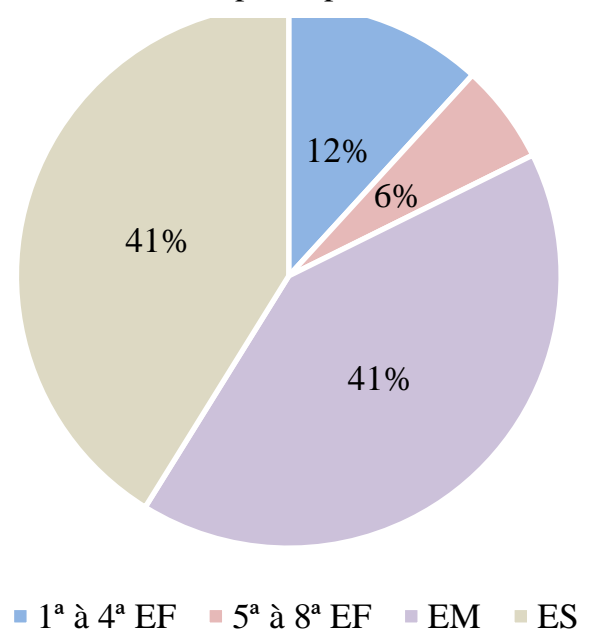

EF: ensino fundamental. EM: ensino médio. ES: ensino superior. Fonte: Autores (2021).

Também foram levantados dados acerca da renda do chefe da família (Figura 2), sendo caracterizado como aquele que recebia o maior salário. Obtivemos como mínimo (1) "Nenhuma renda" e máximo (6) "De 12 a 15 salários-mínimos", com média 3,06 $\pm 1,56$, em avaliação da frequência, obtivemos os seguintes dados: $11,8 \%$ não recebe nenhuma renda, $29,4 \%$ recebem até 1 salário-mínimo, 29,4\% recebem de 1 a 3 salários-mínimos, 11,8\% recebem de 3 a 6 salários-mínimos, 11,8\% recebem de 6 a 9 salários-mínimos e 5,9\% recebem de 12 a 15 salários-mínimos.

Na análise dos escores do SF-36 (Figura 3) é possível observar que três domínios apresentaram uma pontuação média 
menor que 49, classificado como inferior, sendo eles: DOR: 37,76 e LAE: 41,16. Dentre os domínios com escore mediano (5074), podemos visualizar: CF: 73,24; LAF: 61,76; EGS: 57,59; VIT: 50,59; AS: 62,5 e SM: 54,12. Não foi possível observar nenhum domínio com escore médio superior.

Na Tabela 1 também denotamos que nos domínios DOR, 13 indivíduos estavam classificados no escore inferior e no domínio LAE, 11 indivíduos classificados em escore inferior.

Foi ainda questionado aos participantes do estudo, durante realização da Anamnese, se eles se consideravam ansiosos (Figura 4), onde $88 \%$ dos participantes relataram se sentir ansiosos $(n=15)$ e apenas $12 \%$ da amostra relataram não se sentir ansiosa $(\mathrm{n}=2)$ durante o período de isolamento social.

A partir da avaliação do Recordatório 24 Horas, também foi possível observar os seguintes dados sobre o consumo dos participantes (Tabela 2): Ingesta de Açúcares e Doces, Óleos e Gorduras, Frutas, Legumes e Verduras, grupos alimentares reconhecidamente relacionados a sintomas de ansiedade e depressão, buscando assim realizar uma correlação entre os níveis de consumo destes grupos alimentares, com a descrição da percepção de ansiedade apontado pelos participantes.

Figura 2. Renda do chefe de família avaliada em salários. Olinda, PE, Brasil, 2020.

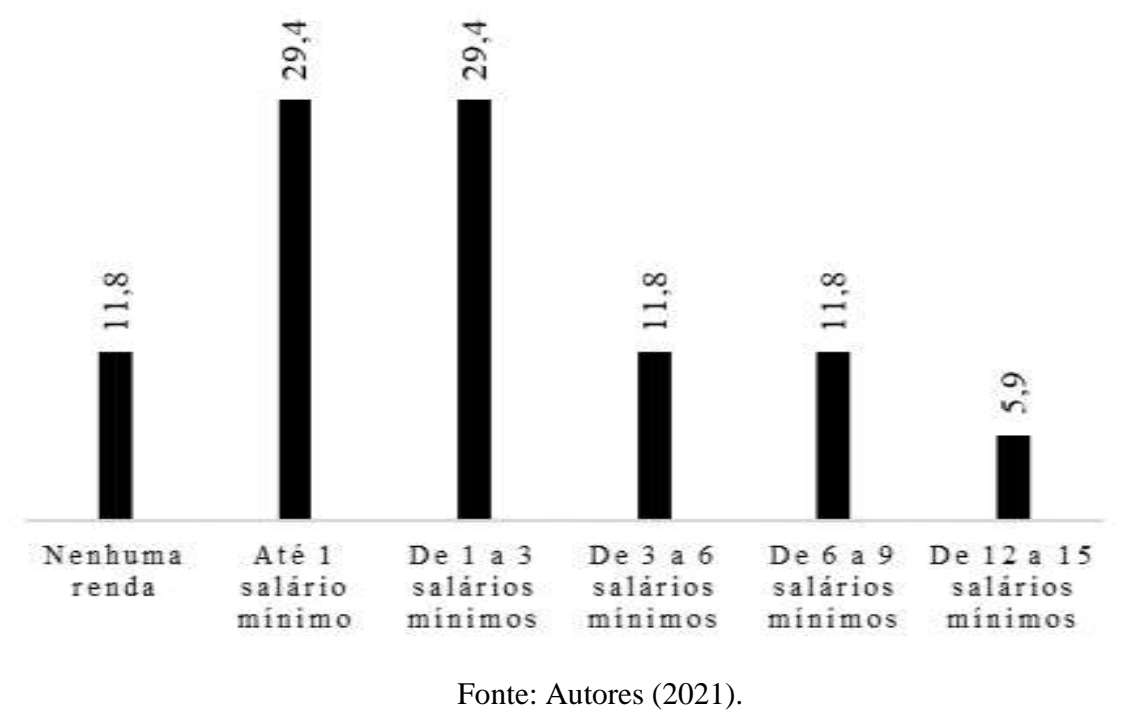

\section{Discussão}

A avaliação da qualidade de vida se tornou algo extremamente relevante nas últimas décadas, onde esta estratégia foi inicialmente adotada por outras áreas do conhecimento, posteriormente incorporada à área da saúde, sendo então denominada qualidade de vida relacionada à saúde. Com isto, se tornou indispensável realizar a mudança da medida, que antes era subjetiva e agora, torna-se quantitativa, onde este resultado seria útil em diversas populações, assim como para diversas doenças (Scheicher, 2002). 
Figura 3. Distribuição das médias dos Scores do SF-36. Olinda, PE, Brasil, 2020.

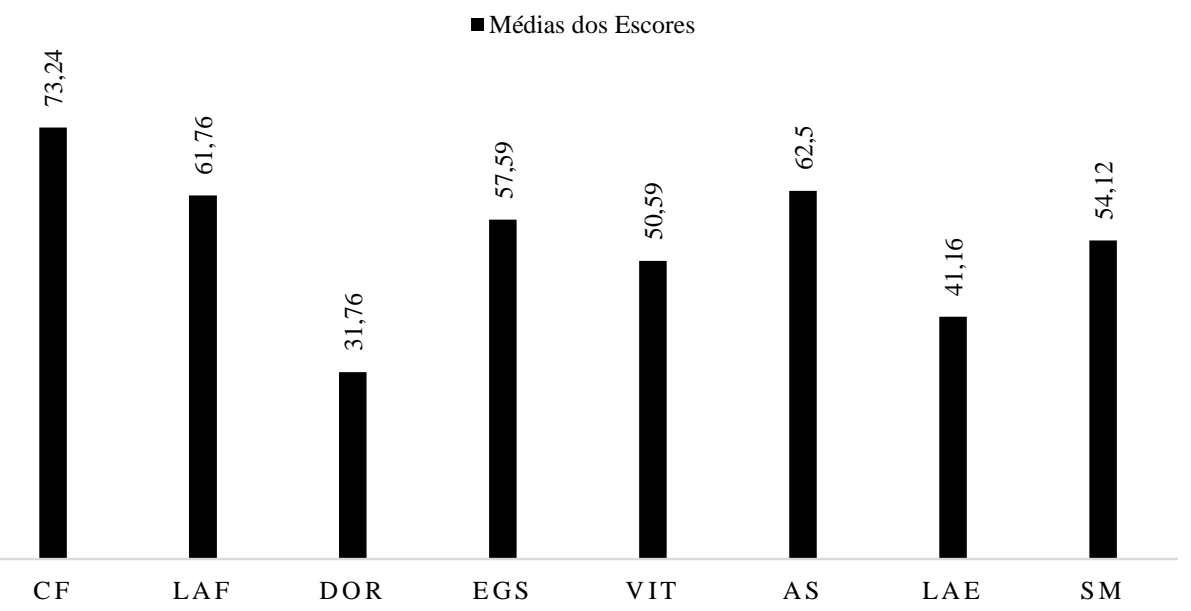

CF: capacidade funcional. LAF: limitações por aspectos físicos. DOR: dor. EGS: estado geral de saúde. VIT: vitalidade. AS: aspectos sociais. LAE: limitações por aspectos emocionais. SM: saúde mental. FR: Frequência. \%: percentual. Fonte: autores (2021).

Figura 4. Percepção de ansiedade autoavaliada pelos participantes. Olinda, PE, Brasil, 2020.

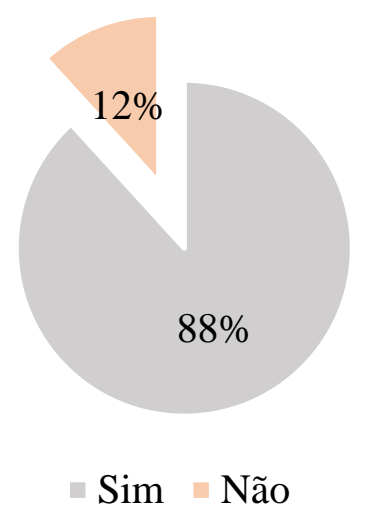

Fonte: Autores (2021).

Tabela 1. Avaliação de frequência e porcentagem dos escores do questionário sf-36. Olinda, PE, brasil, 2020.

\begin{tabular}{cccccccc}
\hline & \multicolumn{2}{c}{ Escore inferior $\mathbf{( 0 - 4 9 )}$} & \multicolumn{2}{c}{ Escore mediano (50-74) } & Escore superior (75-100) \\
\hline Domínios & FR & \% & FR & \% & FR & \% \\
\hline CF & 2 & 11,8 & 5 & 29,5 & 10 & 11 & 68,9 \\
LAF & 6 & 35,3 & 0 & 0 & 0 & 0 & 17,7 \\
DOR & 13 & 76,5 & 4 & 23,5 & 3 & 11,8 \\
EGS & 5 & 29,4 & 9 & 52,9 & 2 & 47,1 \\
VIT & 6 & 35,3 & 9 & 52,9 & 8 & 29,4 \\
AS & 6 & 35,3 & 3 & 17,7 & 5 & 17,7 \\
LAE & 11 & 64,7 & 1 & 5,9 & 3 & 3,3 & 3
\end{tabular}

CF: capacidade funcional. LAF: limitações por aspectos físicos. DOR: dor. EGS: estado geral de saúde. VIT: vitalidade. AS: aspectos sociais. LAE: limitações por aspectos emocionais. SM: saúde mental. FR: Frequência. \%: percentual. Fonte: Autores (2021). 
Tabela 2. Consumo médio de grupos da Pirâmide Alimentar. Olinda, PE, Brasil,

\begin{tabular}{ll}
\hline GRUPO ALIMENTAR & Porções Por Dia \\
\hline Açúcares e doces & 1,64 porção diária \\
Óleos e gorduras & 0,5 porção diária \\
Frutas & 1,3 porção diária \\
Legumes e verduras & 1 porção diária \\
\hline
\end{tabular}

Fonte: Autores (2021).

Dentre os diversos fatores que podem impactar sobre a qualidade de vida, a escolaridade é uma variável importante. Durante a aplicação do SF-36, em um estudo realizado no Reino Unido, 98\% dos participantes conseguiram completar a pesquisa, quantitativo que se aproxima do presente estudo, onde $100 \%$ dos indivíduos conseguiram completar o questionário. Este fator apresenta grande relação com o nível de escolaridade da população estudada, onde o fato do público do nosso estudo ser majoritariamente escolarizado pode ter gerado uma melhor compreensão do questionário e assim facilitando a aplicação do mesmo via mídias digitais (Lyons, 1994).

O presente estudo revela maior comprometimento dos domínios dor e limitações por aspectos emocionais, corroborando com os dados encontrados em um estudo realizado por Barros et al., 2020, onde foi avaliado a prevalência do relato de tristeza/depressão, nervosismo e ansiedade. Ficou evidente que durante a pandemia houve elevação de 40,4\% de pessoas tristes ou deprimidas, 52,6\% frequentemente ansiosos ou nervosos, 43,5\% de problemas de sono relacionados à ansiedade e $48 \%$ de problemas de sono preexistentes agravados, indicando que são necessárias maiores provisões de serviços de atenção à saúde mental adaptador ao contexto pandêmico (Barros et al., 2020).

O resultado do nosso estudo demonstra ainda que o domínio dor foi o mais afetado, acarretando um prejuízo latente na qualidade de vida dos indivíduos, podendo este ser um resultado de mudanças dos hábitos dos indivíduos, onde a maioria passou a realizar trabalhos em home-office de maneira inadequada. Tal fato, como aponta o relatório técnico realizado pelo Institute of Employment Studies (IES) realizado em junho/julho, revelou que 57,1\% da amostra relatou dores leves a intensas no pescoço, 51,9\% tiveram dores nos ombros e 58,2\% referiram dores nas costas, sendo necessário o desenvolvimento de estratégias para o acompanhamento destes trabalhadores, de forma a evitar o agravamento do quadro e promovendo a melhoria da qualidade de vida (Ogata et al., 2020).

Dentre os domínios com escore mediano, podemos evidenciar que domínios como a vitalidade, saúde mental e estado geral de saúde também se mostraram prejudicados, tendo como média: 50,59; 54,12 e 57,59, respectivamente. Estes resultados podem estar relacionados diretamente com o fato de que relações interpessoais foram abaladas durante o período de isolamento social, em que os indivíduos se viram obrigados a se distanciarem de seu círculo de amizade. Tal aspecto também pode estar diretamente relacionado ao estado geral de saúde, demonstrando que a saúde emocional representa uma grande parcela do bem-estar e da qualidade de vida dos indivíduos (Lima, 2020).

É válido ressaltar que nenhum dos domínios apresentou média maior que 75 , sendo todos os resultados enquadrados como escore inferior ou mediano onde, dentre eles, a capacidade funcional, relacionada à prática de atividades corriqueiras como: tomar banho, andar vários quarteirões, ou subir um lance de escada, apresentou a maior média entre os domínios, 73,24, demonstrando que apesar do isolamento social, os indivíduos, em geral, não reportaram dificuldades na realização destas atividades.

É possível observar ainda, a partir da análise do R24h dos participantes, que o consumo de frutas, legumes e verduras se encontra abaixo do recomendado, com consumo médio de 1,3 e 1 porção diária, respectivamente, onde de acordo com a Pirâmide Alimentar Brasileira, deve ser realizado o consumo mínimo de 3 porções para cada um dos grupos. Associado a isto, 
nota-se que o consumo de alimentos açucarados se encontra levemente acima dos parâmetros estabelecidos, onde a pirâmide alimentar preconiza consumo de até 1 porção diária (Ministério da Saúde, 1997).

Em estudo realizado por Penaforte et al. (2020), foi destacado o papel que os alimentos ricos em açúcar, associados à baixo consumo de frutas e vegetais, representam na ansiedade, podendo este ser um fator comprometedor da saúde emocional da amostra, que apresentou elevados níveis de ansiedade, fator ainda mais agravado quando levamos em consideração o momento atual vivenciado pela população, que já se encontra emocionalmente debilitada. Penaforte et al., ressalta ainda que o consumo de frutas, legumes e verduras se apresentou como um fator protetor para agravamento da ansiedade, onde o consumo desses grupos levou a um menor desenvolvimento de quadros de ansiedade (Penafort et al., 2020).

Alguns estudos também ressaltam a associação das DCNT com alguns fatores de risco, exacerbados devido ao processo de globalização, como o aumento do tabagismo, níveis elevados de colesterol, aumento da obesidade causada pelo sedentarismo e principalmente, baixo consumo de alimentos considerados saudáveis, como frutas, verduras e legumes, fato este observado também na população do nosso estudo, composta por indivíduos portadores de DCNT (Claro et al., 2013).

A partir da metodologia proposta neste artigo, vale ressaltar a necessidade de medidas de atendimento, acompanhamento e assistência remota do público ambulatorial, principalmente quando se trata de população de risco como idosos, imunossuprimidos, portadores de doenças crônicas. Espera-se a evolução e aplicabilidade da telemedicina e novos modelos de cuidado remoto (Sampa et al, 2020; Bokolo, 2021).

\section{Considerações Finais}

Os dados encontrados no presente estudo demonstraram que grande parte dos indivíduos avaliados apresentam uma percepção de baixa a moderada quando considerada a qualidade de vida durante o isolamento social. Tal processo pode ter refletido diretamente na saúde física, mental e emocional da população, especialmente quando consideradas as medidas restritivas adicionais impostas à indivíduos portadores de DCNT. Além disso, também é possível observar que grande parcela da amostra relatou se sentir ansiosa, evidenciando o desgaste mental sofrido durante este período, agravado ainda mais pelos maus hábitos alimentares da população, que não conta com consumo adequado de importantes grupos de alimentos para a regulação de processos metabólicos responsáveis pelo equilíbrio da saúde e bem-estar. Por fim, ressaltamos que a realização da avaliação da qualidade de vida da população se faz necessária para que se possa investigar melhor os impactos causados pela pandemia do Covid-19 no bem-estar geral da população, em especial à população mais afetada por este processo, que foram os indivíduos portadores de DCNT caracterizados como grupo de risco, destacando que as mídias digitais podem ser um meio seguro para esta avaliação. Trabalhos futuros deverão investigar o impacto a longo prazo da pandemia em portadores de doenças crônica não transmissíveis e novas técnicas de cuidado remoto para atender a população de risco.

\section{Referências}

Barros, M. B. A., Lima, M. G., Malta, D. C., Szwarcwald, C. L., Azevedo, R. C. S., Romero, D., \& Souza, P. R.B. J. (2020). Relato de tristeza/depressão, nervosismo/ansiedade e problemas de sono na população adulta brasileira durante a pandemia do Covid-19. Revista Epidemiologia e Serviços de Saúde, 29(4), e2020427- e2020439.

Bokolo, A. J. (2021). Exploring the adoption of telemedicine and virtual software for care of outpatients during and after COVID-19 pandemic. Irish Journal Of Medical Science, 190(1), 1-10.

Brasil, Ministério da Saúde. (1997). Pirâmide Alimentar Brasileira. Brasília.

Claro, R. M., Santos, M. A. S., Oliveira, T. P., Pereira, C. A., Szwarcwald, C. L., \& Malta, D. C. (2013). Consumo de alimentos não saudáveis relacionados a doenças crônicas não transmissíveis no Brasil: Pesquisa Nacional de Saúde. Revista Epidemiologia e Serviços de Saúde, 24(2), 257-265.

Dempster, M. \& Donnelly, M. (2000). How well do elderly people complete individualized quality of life measures: an exploratory study. Quality of Life Research, 9(4), 369-375.

Fletcher, A. (1995). Economic of hypertension control. Bull WHO, 73(4), 417-24. 
Lima, R. C. (2020). Distanciamento e isolamento sociais pela Covid-19 no Brasil: impactos na saúde mental. Revista Ciência \& Saúde Coletiva, 30(2), e300214- e300224.

Lyons, R. A., Perry, H. M. \& Littlepage, B. N. (1994). Evidence for the validity of Short-Form 36 Questionnaire (SF-36) in an elderly population. Age and Ageing, 23(3), 182-184.

Mahony, P. G., Rodgers, H., Thomson, R. G., Dobson, R. \& James, O. F. (1998). Is the SF-36 suitable for assessing health status of older stroke patients? Age and Ageing, 27(1), 19-22.

Ogata, A. J. N., Pinto, A. C., Pereira, V. S., Lourenço, V., Andrade, Y. \& Malik, A. M. (2020). Relatório técnico I: Impacto na saúde e no bem-estar do trabalho em regime de home-office durante a pandemia do SARD-COVID-19.

Penaforte, F. R. O., Matta, N. C. \& Japur, C. C. (2020). Association between stress and eating behavior in college students. Demetra, $11(1), 225-237$.

Pierin, A. M. G. (2004). Hipertensão arterial uma proposta para o cuidar. Manole.

Sampa, M. B., Hoque, M. R., Islam, R., Nishikitani, M., Nakashima, N., Yokota, F., Kikuchi, K., Rahman, M. M., Shah, F. \& Ahmed, A. (2020). Redesigning Portable Health Clinic Platform as a Remote Healthcare System to Tackle COVID-19 Pandemic Situation in Unreached Communities. International Journal Of Environmental Research And Public Health, 17(13), 4709-4723.

Scheicher, M.E. (2002). Qualidade de vida e função cardíaca no infarto do miocárdio. Revista de fisioterapia da Universidade de São Paulo, 9(2), 63-8.

Silva, D. M. G. V., Souza, S, S., Francioni, F. F. \& Meirelles, B. H. S. (2005). Qualidade de vida na perspectiva de pessoas com problemas respiratórios crônicos: a contribuição de um grupo de convivência. Revista Latino-Americana de Enfermagem, 13(1), 7-14.

Somchida, A. \& Fernandes, F. C. (2003). Saúde e qualidade de vida na terceira idade: uma introspecção dos idosos institucionalizados. Associação Brasileira de Odontologia, 1(1).

Taylor, S. E. (1999). Health psychology (4a ed). Mc GrawHill.

Testa, M. A. \& Simonson, D. C. (1996). Assessment of quality of life outcomes. The New England Journal of Medicine, 334(13), 835-840.

Thomas, J. R., Nelson, J. K. \& Silverman, S. J. (2012). Métodos de pesquisa em atividade Física. (6a ed).

Walters, S. J., Munro, J. F. \& Brazier, J. E. (2001). Using the SF-36 with older adults: a cross-sectional communitybased survey. Age and Ageing, 30(4), 337343. 